\title{
Volumetric Assessment of Corticospinal Tract Infiltration by Astrocytoma Using Diffusion Tensor Tractography
}

\author{
Marwa Adel Shaaban', Taha Kamel Alloush2, Mohamed Hamdy Ibrahim², \\ Ahmed Abd el bar ${ }^{3}$, Sherif Hashim Morad ${ }^{3}$ \\ ${ }^{1}$ Department of Radiology, Misr University for Science and Technology, Cairo, Egypt \\ ${ }^{2}$ Department of Neurology, Ain Shams University, Cairo, Egypt \\ ${ }^{3}$ Department of Neurosurgery, Ain Shams University, Cairo, Egypt \\ Email: mohamedhamdy neuro2007@yahoo.com
}

Received 12 April 2014; revised 23 May 2014; accepted 30 May 2014

Copyright (C) 2014 by authors and Scientific Research Publishing Inc.

This work is licensed under the Creative Commons Attribution International License (CC BY).

http://creativecommons.org/licenses/by/4.0/

(c) (i) Open Access

\begin{abstract}
Objectives: The aim of the study is to assess volumetric Corticospinal Tract infiltration by Astrocytoma using Diffusion Tensor Tractography. Material and Methods: Preoperative, anatomic (T1and T2-weighted) and diffusion tensor MRI were performed in 9 patients with supratentorial gliomas (WHO grade II and III). The tumors were manually segmented from the T1- and T2-weighted MRI, and their volume calculated. A three-dimensional tractography was performed in each case. A second segmentation and volume measurement was performed on the tumor regions intersecting adjacent white matter fiber tracts. Results: We identified that white matter tracts were infiltrated by 6 Astrocytoma tumors. The median tumor volume ( \pm standard deviation) in our patient population was $33 \pm 26.82 \mathrm{ml}$. The median tumor volume ( \pm standard deviation) infiltrating white matter fiber tracts was $4.15 \pm 9.23 \mathrm{ml}$. The median fraction of tumor volume infiltrating white matter fiber tracts was $26.3 \% \pm \mathbf{1 0 . 1 \%}$. Conclusions: Diffusion tensor MR Tractography is a reliable preoperative assessment tool since it detects extensive white matter infiltration by Astrocytoma irrespective of brain tumors volume. Recommendations: Prospective large population studies are required to clarify how infiltration relates to tumor location.
\end{abstract}

\section{Keywords}

Tractography, Astrocytoma, Brain Tumor, Corticospinal Tract Infiltration 


\section{Introduction}

The survival of patients harboring primary brain tumors appears to be influenced by a variety of factors, such as age, histologic tumor type, gender, preoperative Karnofsky performance score, epilepsy as presenting symptom, tumor involvement of the contralateral hemisphere, and extent of tumor resection [1]-[3]. Surgical resection plays an important role in the management of both low- and high-grade primary brain tumors. Although no prospective randomized outcome study has been conducted to date, retrospective data suggest an association between gross total tumor resection and increased overall survival [4]-[6].

The goal of surgical treatment is to remove as much tumor tissue as possible, while in the same time preserving the integrity of functionally eloquent gray and white matter structures, and thus avoids postoperative neurologic deficits. However, tumor infiltration of eloquent cortical areas and/or white matter tracts may preclude safe gross total resection. Consequently, knowledge of the relationship between tumor and eloquent cortical and white matter regions might be helpful for preoperatively determining the extent to which a brain tumor can be surgically removed, and also for guiding the actual surgical procedure.

Diffusion magnetic resonance (MR) imaging is evolving into a potent tool in the examination of the central nervous system. Although it is often used for the detection of acute ischemia, evaluation of directionality in a diffusion measurement can be useful in white matter, which demonstrates strong diffusion anisotropy. Techniques such as diffusion-tensor imaging offer a glimpse into brain microstructure at a scale that is not easily accessible with other modalities, in some cases improving the detection and characterization of white matter abnormalities. Diffusion MR tractography offers an overall view of brain anatomy, including the degree of connectivity between different regions of the brain. However, optimal utilization of the wide range of data provided with directional diffusion MR measurements requires careful attention to acquisition and post processing [7] [8].

Based on DTI findings, resulting from studies of brain tumor patients, the white matter involvement by a tumor can be arranged into three different categories:

- Displaced: maintained normal anisotropy relative to the contralateral tract in the corresponding location, but situated in an abnormal T2-weighted signal intensity area or presented an abnormal orientation.

- Infiltrated: reduced anisotropy but remaining identifiable on orientation maps.

- Edematous: maintained normal anisotropy and normally oriented but located in an abnormal T2-weighted signal intensity area [9] [10].

\section{Materials and Methods}

\subsection{Patient Population}

The study was done between October 2012 and December 2013 where 33 patients with intracranial space-occupying lesions underwent surgery. Patients were informed about study objectives and hazards and written consent were obtained. Ethical committee approved the study.

Among them, nine patients had lesions near the corticospinal tract, on the basis of conventional MR imaging findings, and these nine patients (five men, four women; mean age, 43 years; age range, 29 - 62 years) were included in our study. Six patients were ASTROCYTOMA WHOII. All the patients were admitted to our institution for neurosurgery, and routine preoperative evaluations were performed, which included 1-T MR imaging. Full neurological examinations were performed (Table 1), and consequently all patients in this series underwent preoperative diffusion tensor MRI (DT-MRI) as part of surgical planning for image-guided tumor resection, in addition to preoperative anatomic T2-weighted fast spin echo (T2FSE), and volumetric T1-weighted MRI (3DSPGR). All patients in this series underwent image-guided tumor resection. Histopathologic examination of the ressected tumor tissue confirmed the diagnosis of low- or high-grade glioma in each case.

\subsection{Image Acquisition}

All imaging studies were performed on Philips open MRI-scanner, with field strength of 1 Tesla (Philips Panorama). Between 2 and 7 days before surgery, DT imaging and anatomic T1-weighted volume imaging were performed with a 40-mT/m gradient. DT imaging was performed by using a single-shot spin-echo echo-planar MR sequence with the following parameters: 5200/79 (repetition time msec/echo time msec), with a motion-probing gradient in 12 noncolinear directions; $b$ value, $700 \mathrm{sec} / \mathrm{mm}^{2}$; matrix, $128 \times 128$; voxel size, $1.7 \times 1.7 \times 3 \mathrm{~mm}$; no intersection gap; and four signals acquired. The generalized auto calibrating partial parallel acquisition algorithm 
Table 1. Patient population and tumor characteristics.

\begin{tabular}{ccccc}
\hline $\begin{array}{c}\text { Case } \\
\text { No. }\end{array}$ & $\begin{array}{c}\text { Sex, Age } \\
\text { (yrs.) }\end{array}$ & Tumor location & Histopathology & $\begin{array}{c}\text { Eloquent cortical and white matter areas } \\
\text { affected }\end{array}$ \\
\hline $\mathbf{1}$ & M 32 & L frontal & Astrocytoma WHO II & SMA, motor strip, motor pathway \\
2 & F 34 & R temporal & Oligodendroglioma WHO II & Wernicke's area, optic radiation \\
$\mathbf{3}$ & F $\mathbf{4 1}$ & L Tempro-parietal & Astrocytoma WHO II & $\begin{array}{c}\text { SMA, motor pathway } \\
4\end{array}$ \\
M $\mathbf{6 1}$ & R frontal medial & Anaplasic Astrocytoma & Motor strip, motor pathway \\
$\mathbf{5}$ & M $\mathbf{3 8}$ & L fronto-temporal & Astrocytoma WHO II & Motor strip, motor pathway \\
$\mathbf{6}$ & F $\mathbf{4 5}$ & L frontal & Anaplastic strocytoma & $\begin{array}{c}\text { Motor and sensory strip, motor pathway, } \\
\text { arcuate (superior longitudinal) fasciculus }\end{array}$ \\
7 & F 46 & R occipital & Oligodendroglioma WHO II & Optic radiation \\
8 & M 34 & L insular & Ganglioglioma & Motor pathway, uncinate fasciculus \\
$\mathbf{9}$ & M $\mathbf{2 8}$ & R frontal & Anaplastic Astrocytoma (WHO III) & Motor strip, motor pathway \\
\hline
\end{tabular}

Careful analysis of the table showed that $66.6 \%$ (4/6) Astrocytoma tumors are Frontal in position and one case is frontotemporal and the last one is tempro-parietal.

was applied for parallel imaging, with a reduction factor of two, an additional 24 autocalibrating phase-encoding lines in the center of k-space, and a 75\% partial Fourier technique in the phase-encoding direction. A total of 40 transverse sections were obtained to cover the whole brain. Imaging time was 7 minutes 20 seconds. A magnetization-prepared rapid acquisition gradient-echo sequence was applied for anatomic T1-weighted volume MR data acquisition by using the following parameters: 2000/4.4/990 (repetition time msec/echo time msec/inversion time msec); matrix, $256 \times 240$; voxel size, $0.9 \times 0.9 \times 1 \mathrm{~mm}$; and two signals acquired. The generalized auto calibrating partial parallel acquisition algorithm was also applied with a reduction factor of two for the magnetization-prepared rapid acquisition gradient-echo sequence. A total of 208 transverse sections were obtained to cover the whole brain without intersection gaps. Field center was equivalent between the two sequences in each patient. Diffusion tensor imaging: axial and coronal line scan diffusion images (TE $=64 \mathrm{msec}$, TR $=2592 \mathrm{msec}$, slice thickness $4 \mathrm{~mm}$ ) were acquired, covering the entire tumor and adjacent healthy brain parenchyma, as well as the brainstem and basal ganglia regions. Each section from the DT-MRI sequence was acquired from six non-colinear gradient directions. In addition, a baseline T2-weighted slice was acquired for each section.

\subsection{Image Analysis}

The 3D-Slicer software package [11]-[15] was used for image registration and processing. This is a modular software package with image registration, segmentation and DT-MRI processing capabilities.

First, the anatomic (3D-SPGR and T2-FSE) and the DT-MRI scans were rigidly registered, using a maximization of mutual information (MI) algorithm.

This algorithm is fully implemented in the 3D-Slicer software package, and it is known to provide robust and accurate results [16] [17]. Manual adjustments were made where necessary, using as reference anatomic landmarks such as the anterior and posterior commissures, lateral ventricles, and corpus callosum.

Each tumor was manually segmented (outlined) from the anatomic T2-FSE scans using the 3D-Slicer and a computer mouse. According to the literature, among anatomic MRI modalities, T2-weighted images appear to reflect the tumor extent more closely, although occult tumor can be found even beyond the region of increased T2-signal in some gliomas [18]-[22]. The tumor volume was computed from the segmented area and voxel size, using the 3D-Slicer software.

In the next step, the white matter structure from DT-MRI was visualized. First, fractional anisotropy maps were generated in each case, using the 3D-Slicer software. Next, we obtained both a 2D-visualization [23] and a 3D-tractography in each patient. For the 3D-tractography, we used the algorithm described by Westin et al. [24], which is fully implemented in the 3D-Slicer package. In order to calculate the trajectory of fiber tracts, this algorithm takes into account fractional anisotropy, as well as the angle between the primary eigenvector within a voxel and the equivalent vector in the neighboring voxels. In order to selectively visualize each fiber tract expected to be in close spatial relationship with the tumor (based on anatomic MRI), we defined seed points, from 
which the tractography was initialized: ventral brainstem for reconstructing the corticospinal tract, the white matter region adjacent to the lateral geniculate body for the optic radiation, the temporal lobe stem for tracking the uncinate fasciculus, etc. The 3D-tractography results were compared with the corresponding 2D-visualization maps and manually corrected, in order to insure that artifacts were removed.

Finally, the tumor regions intersecting white matter tracts were manually segmented and their volume calculated in the same manner as the total tumor volume.

\section{Results}

From the 9 cases of tumors in relation to corticospinal tract, we identified that white matter tracts were infiltrated by 6 Astrocytoma tumors. With respect to Table 2 the median tumor volume ( \pm standard deviation) in our patient population was $33 \pm 26.82 \mathrm{ml}$. The median tumor volume ( \pm standard deviation) infiltrating white matter fiber tracts were $4.15 \pm 9.23 \mathrm{ml}$. The median fraction of tumor volume infiltrating white matter fiber tracts was $26.3 \% \pm 10.1 \%$.

Careful analysis of the table showed that the there is difference in tumor behaviour apart from its gross volume. As in case number 4 where Tumor volume is $9.3 \mathrm{ml}$ however $28 \%$ of its volume is infiltrating the tract (Figure 1) and if compared to case number 9 where tumor volume is $91.8 \mathrm{ml}$ (larger than case 4 ) yet only 24.6\% are infiltrating the tract. Also case number 6 has highest fraction of tumor volume infiltrating the corticospinal

Table 2. Volumetric assessment of white matter infiltration.

\begin{tabular}{cccc}
\hline $\begin{array}{c}\text { Case } \\
\text { No. }\end{array}$ & $\begin{array}{c}\text { Tumor volume } \\
(\mathbf{m l})\end{array}$ & $\begin{array}{c}\text { Tumor volume infiltrating fiber tracts } \\
(\mathbf{m l})\end{array}$ & $\begin{array}{c}\text { Fraction of Tumor volume infiltrating fiber tracts } \\
\text { (\% of total tumor volume) }\end{array}$ \\
\hline $\mathbf{1}$ & $\mathbf{3 3 . 7}$ & $\mathbf{4 . 8}$ & $\mathbf{1 4 . 2}$ \\
2 & 51.3 & 13.7 & 26.7 \\
$\mathbf{3}$ & $\mathbf{3 2 . 3}$ & $\mathbf{2 . 4}$ & $\mathbf{7 . 4}$ \\
$\mathbf{4}$ & $\mathbf{9 . 3}$ & $\mathbf{2 . 6}$ & $\mathbf{2 8 . 0}$ \\
$\mathbf{5}$ & $\mathbf{2 8 . 3}$ & $\mathbf{3 . 5}$ & $\mathbf{1 2 . 4}$ \\
$\mathbf{6}$ & $\mathbf{6 2 . 6}$ & $\mathbf{2 3 . 1}$ & $\mathbf{3 6 . 9}$ \\
7 & 9.2 & 2.6 & 28.3 \\
8 & 13.0 & 2.0 & 15.4 \\
$\mathbf{9}$ & $\mathbf{9 1 . 8}$ & $\mathbf{2 2 . 6}$ & $\mathbf{2 4 . 6}$ \\
\hline
\end{tabular}
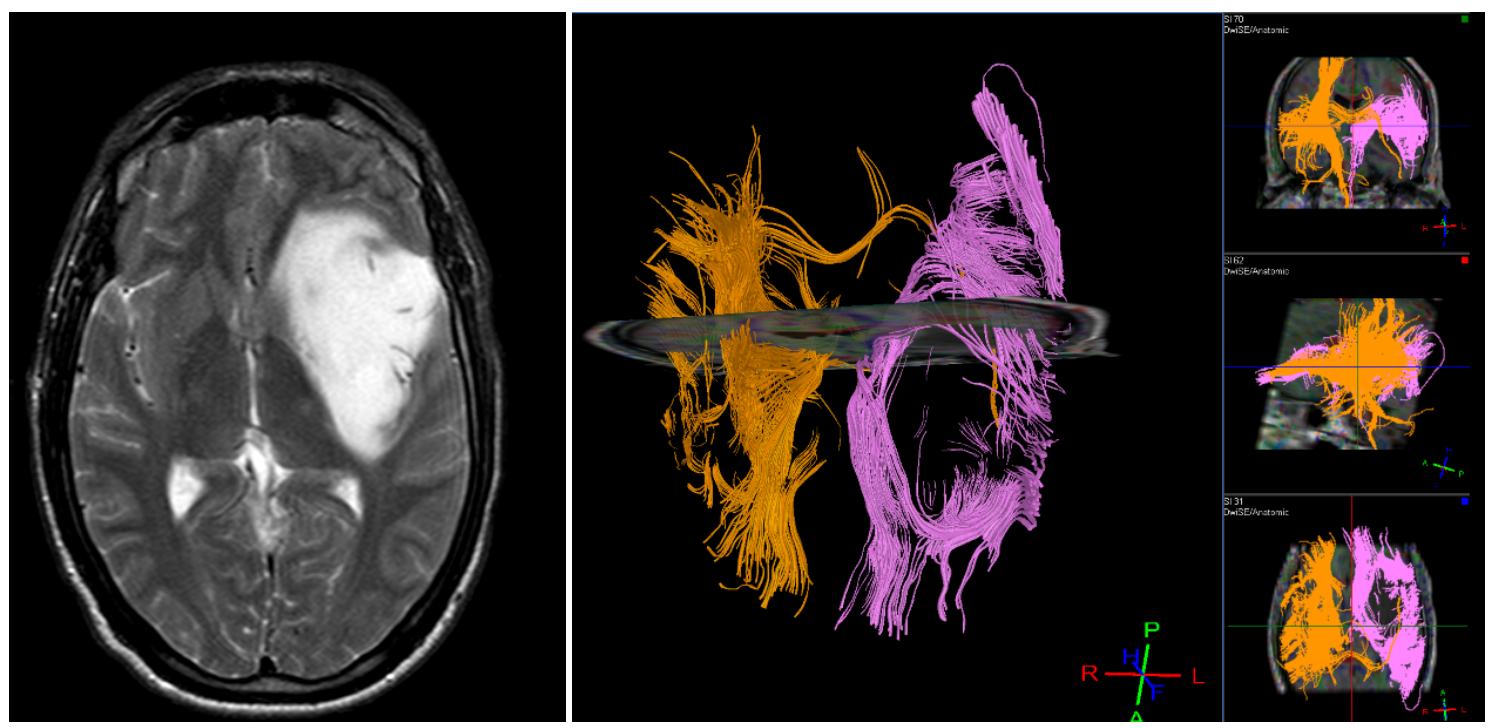

Figure 1. Left Insular Astrocytoma in a 33 years old male. Tractography shows infiltration of the CST. 
tract. So the fraction of tumor volume infiltration is not directly proportional to the tumor size. This is an interesting point needs further study.

\section{Discussion}

Intracranial space-occupying lesions located in the eloquent brain area remain a challenge from the perspective of surgical removal with preservation of brain function. Image-guided neurosurgery is a tool that can help to prevent damage to the eloquent cortical and white matter areas [25] [26].

In the pre-DT-MRI era, some intraoperative white matter stimulation studies suggested that preserved white matter tracts may be present within some gliomas [27]. The advent of DT-MRI has enabled in vivo, non-invasive studies of tumor-induced white matter changes [10]-[13], and patterns of tumor invasion [14]. Furthermore, DTMRI is being increasingly used for guiding surgical procedures [28]. We have shown that, by combining the information provided by anatomic and diffusion tensor MRI, it is possible to quantify the extent of tumor infiltration of adjacent white matter. Such a quantitative measure may help improve the accuracy of currently available predictive models for brain tumor resectability [29].

The information provided by DT-MRI can potentially help to further improve surgical outcomes of brain tumor patients, and we illustrated this point at the example of two cases from our series.

Results of our study: As regard, Astrocytoma showed that the median tumor volume ( \pm standard deviation) in our patient population was $33 \pm 26.82 \mathrm{ml}$. The median tumor volume ( \pm standard deviation) infiltrating white matter fiber tracts was $4.15 \pm 9.23 \mathrm{ml}$. The median fraction of tumor volume infiltrating white matter fiber tracts was $26.3 \% \pm 10.1 \%$. This is slightly different than a study done by Florin and his colleagues 2007 [30] where his study showed that the median tumor volume ( \pm standard deviation) in our patient population was $42.5 \pm 28.9$ $\mathrm{ml}$. The median tumor volume ( \pm standard deviation) infiltrating white matter fiber tract was $5.2 \pm 9.9 \mathrm{ml}$. The median percentage of tumor volume infiltrating white matter fiber tracts was $21.4 \% \pm 9.7 \%$. This slight difference could be related to sample size difference between the 2 studies. During our study we noticed mismatch between total tumor volume and Fraction of Tumor volume infiltrating fiber tracts (\% of total tumor volume) some tumors have larger volume (case 9) however less fraction of tumor volume infiltrating fiber. This suggests that tumors have unpredicted infiltrating behaviour power and Tractography can help in early detection of infiltration.

\section{Limitations}

Our study is limited by the relatively small sample size.

\section{Recommendations}

Prospective, large population studies are required in order to definitively clarify this issue. In addition, studies correlating the imaging findings with histology are necessary, in order to validate our results.

\section{References}

[1] Central Brain Tumor Registry of the United States (2005) Statistical Report: Primary Brain Tumors in The United States 1998-2002. Primary Brain Tumors in The United States 1998-2002.

[2] Nicolato, A., Gerosa, M.A., Fina, P., Iuzzolino, P., et al. (1995) Prognostic Factors in Low-Grade Supratentorial Astrocytomas: A Uni-Multivariate Statistical Analysis in 76 Surgically Treated Adult Patients. Surgical Neurology, 44, 208-221. (Discussion 221-223)

[3] Pignatti, F., Van den Bent, M., Curran, D., Debruyne, C., et al. (2002) Prognostic Factors for Survival in Adult Patients with Cerebral Low-Grade Glioma. Journal of Clinical Oncology, 20, 2076-2084. http://dx.doi.org/10.1200/JCO.2002.08.121

[4] Janny, P., Cure, H., Mohr, M., Heldt, N., et al. (1994) Low Grade Supratentorial Astrocytomas. Management and Prognostic Factors. Cancer, 73, 1937-1945. http://dx.doi.org/10.1002/1097-0142(19940401)73:7<1937::AID-CNCR2820730727>3.0.CO;2-G

[5] Keles, G.E., Lamborn, K.R. and Berger, M.S. (2001) Low-Grade Hemispheric Gliomas in Adults: A Critical Review of Extent of Resection as a Factor Influencing Outcome. Journal of Neurosurgery, 95, 735-745. http://dx.doi.org/10.3171/jns.2001.95.5.0735 
[6] Claus, E.B. and Black, P.M. (2006) Survival Rates and Patterns of Care for Patients Diagnosed with Supratentorial Low-Grade Gliomas: Data from the SEER Program 1973-2001. Cancer, 106, 1358-1363. http://dx.doi.org/10.1002/cncr.21733

[7] Mori, S. and Van Zijl, P.C. (2002) Fiber Tracking: Principles and Strategies-A Technical Review. NMR in Biomedicine, 15, 468-480. http://dx.doi.org/10.1002/nbm.781

[8] Lowe, M.J., Horenstein, C., Hirsch, J.G., Marrie, R.A., et al. (2006) Functional Pathway-Defined MRI Diffusion Measures Reveal Increased Transverse Diffusivity of Water in Multiple Sclerosis. Neuroimage, 32, 1127-1133. http://dx.doi.org/10.1016/j.neuroimage.2006.04.208

[9] Kealey, S.M., Kim, Y., Whiting, W.L., Madden, D.J., et al. (2005) Determination of Multiple Sclerosis Plaque Size with Diffusion-Tensor MR Imaging: Comparison Study with Healthy Volunteers. Radiology, 236, 615-620. http://dx.doi.org/10.1148/radiol.2362040014

[10] Witwer, B.P., Moftakhar, R., Hasan, K.M., Deshmukh, P., et al. (2002) Diffusion-Tensor Imaging of White Matter Tracts in Patients with Cerebral Neoplasm. Journal of Neurosurgery, 97, 568-575. http://dx.doi.org/10.3171/jns.2002.97.3.0568

[11] Mori, S., Frederiksen, K., Van Zijl, P.C., Stieltjes, B., et al. (2002) Brain White Matter Anatomy of Tumor Patients Evaluated with Diffusion Tensor Imaging. Annals of Neurology, 51, 377-380. http://dx.doi.org/10.1002/ana.10137

[12] Inoue, T., Ogasawara, K., Beppu, T., Ogawa, A., et al. (2005) Diffusion Tensor Imaging for Preoperative Evaluation of Tumor Grade in Gliomas. Clinical Neurology and Neurosurgery, 107, 174-180. http://dx.doi.org/10.1016/j.clineuro.2004.06.011

[13] Goebell, E., Paustenbach, S., Vaeterlein, O., Ding, X.Q., et al. (2006) Low-Grade and Anaplastic Gliomas: Differences in Architecture Evaluated with Diffusion-Tensor MR Imaging. Radiology, 239, 217-222. http://dx.doi.org/10.1148/radiol.2383050059

[14] Mandonnet, E., Capelle, L. and Duffau, H. (2006) Extension of Paralimbic Low Grade Gliomas: Toward an Anatomical Classification Based on White Matter Invasion Patterns. Journal of Neuro-Oncology, 78, 179-185. http://dx.doi.org/10.1007/s11060-005-9084-y

[15] Gering, D.T., Nabavi, A., Kikinis, R., Hata, N., et al. (2001) An Integrated Visualization System for Surgical Planning and Guidance Using Image Fusion and an Open MR. Journal of Magnetic Resonance Imaging, 13, 967-975. http://dx.doi.org/10.1002/jmri.1139

[16] Wells 3rd, W.M., Viola, P., Atsumi, H., Nakajima, S., et al. (1996) Multi-Modal Volume Registration by Maximization of Mutual Information. Medical Image Analysis, 1, 35-51. http://dx.doi.org/10.1016/S1361-8415(01)80004-9

[17] West, J., Fitzpatrick, J.M., Wang, M.Y., Dawant, B.M., et al. (1997) Comparison and Evaluation of Retrospective Intermodality Brain Image Registration Techniques. Journal of Computer Assisted Tomography, 21, 554-566. http://dx.doi.org/10.1097/00004728-199707000-00007

[18] Nimsky, C., Fujita, A., Ganslandt, O., Von Keller, B., et al. (2004) Volumetric Assessment of Glioma Removal by Intraoperative High-Field Magnetic Resonance Imaging. Neurosurgery, 55, 358-370. (Discussion 370-1)

[19] Daumas-Duport, C., Varlet, P., Tucker, M.L., Beuvon, F., et al. (1997) Oligodendrogliomas. Part I: Patterns of Growth, Histological Diagnosis, Clinical and Imaging Correlations: A Study of 153 Cases. Journal of Neuro-Oncology, 34, 3759. http://dx.doi.org/10.1023/A:1005707203596

[20] Kelly, P.J., Daumas-Duport, C., Kispert, D.B., Kall, B.A., et al. (1987) Imaging-Based Stereotaxic Serial Biopsies in Untreated Intracranial Glial Neoplasms. Journal of Neurosurgery, 66, 865-874. http://dx.doi.org/10.3171/jns.1987.66.6.0865

[21] Price, S.J., Jena, R., Burnet, N.G., Hutchinson, P.J., et al. (2006) Improved Delineation of Glioma Margins and Regions of Infiltration with the Use of Diffusion Tensor Imaging: An Image-Guided Biopsy Study. American Journal of Neuroradiology, 27, 1969-1974.

[22] Provenzale, J.M., McGraw, P., Mhatre, P., Guo, A.C., et al. (2004) Peritumoral Brain Regions in Gliomas and Meningiomas: Investigation with Isotropic Diffusion-Weighted MR Imaging and Diffusion-Tensor MR Imaging. Radiology, 232, 451-460. http://dx.doi.org/10.1148/radiol.2322030959

[23] Peled, S., Gudbjartsson, H., Westin, C.F., Kikinis, R., et al. (1998) Magnetic Resonance Imaging Shows Orientation and Asymmetry of White Matter Fiber Tracts. Brain Research, 780, 27-33. http://dx.doi.org/10.1016/S0006-8993(97)00635-5

[24] Westin, C.F., Maier, S.E., Mamata, H., Nabavi, A., et al. (2002) Processing and Visualization for Diffusion Tensor MRI. Medical Image Analysis, 6, 93-108. http://dx.doi.org/10.1016/S1361-8415(02)00053-1

[25] Ojemann, J.G., Miller, J.W. and Silbergeld, D.L. (1996) Preserved Function in Brain Invaded by Tumor. Neurosurgery, 39, 253-258. (Discussion 258-259)

[26] Schiffbauer, H., Berger, M.S., Ferrari, P., Freudenstein, D., et al. (2002) Preoperative Magnetic Source Imaging for 
Brain Tumor Surgery: A Quantitative Comparison with Intraoperative Sensory and Motor Mapping. Journal of Neurosurgery, 97, 1333-1342. http://dx.doi.org/10.3171/jns.2002.97.6.1333

[27] Skirboll, S.S., Ojemann, G.A., Berger, M.S., Lettich, E., et al. (1996) Functional Cortex and Subcortical White Matter Located within Gliomas. Neurosurgery, 38, 678-684. (Discussion 684-685)

[28] Nimsky, C., Ganslandt, O. and Fahlbusch, R. (2006) Implementation of Fiber Tract Navigation. Neurosurgery, 58, ONS292-ONS304.

[29] Talos, I.F., Zou, K.H., Ohno-Machado, L., Bhagwat, J.G., et al. (2006) Supratentorial Low-Grade Glioma Resectability: Statistical Predictive Analysis Based on Anatomic MR Features and Tumor Characteristics. Radiology, 239, 506-513. http://dx.doi.org/10.1148/radiol.2392050661

[30] Talos, I.F., Zou, K.H., Kikinis, R. and Jolesz, F.A. (2007) Volumetric Assessment of Tumor Infiltration of Adjacent White Matter Based on Anatomic MRI and Diffusion Tensor Tractography. Academic Radiology, 14, 431-436. 